\title{
BACTERIOLOGIC STUDIES IN ACUTE RHEUMATIC FEVER *
}

\author{
HOMER F. SWIFT, M.D., AND RALPH A. KINSELLA, M.D. \\ NEW YORK
}

The present study was undertaken to determine whether any constant cultural or immunologic type of bacterium was associated with acute rheumatic fever. This disease presents a fairly typical clinical picture, consisting of an acute febrile condition, associated with polyarthritis, and a tendency to involvement of such serous surfaces as the endocardium, pericardium and pleura. Muscular tissues are also frequently involved, most noticeably the myocardium, in which a peculiar pathologic picture is commonly found in fatal cases. Certain symptoms of the disease, namely, the fever and polyarthritis, respond in a fairly characteristic manner to the administration of the salicylates in sufficient doses. It does not seem unreasonable, therefore, to suppose that the disease may be the result of the action of some definite pathogenic micro-organism.

The early contention of Achalme ${ }^{1}$ that the disease was due to a gram-negative anaerobic bacillus has been disproved. The next view was that of Singer, ${ }^{2}$ who claimed that the disease was an attentuated pyemia, due most frequently to the streptococci, but also to the staphylococci and other pyogenic organisms. Singer's contentions were based on bacteriologic studies of postmortem material and the isolation of pyogenic cocci from the urine. The sources of error connected with such studies are even more obvious today than they were to his opponents at that time. The extensive studies of Westphal, Wassermann and Malkoff ${ }^{3}$ with a streptococcus which they isolated in a case of rheumatic fever, chorea and hyperpyrexia proved that a nonsuppurative polyarthritis could be produced in rabbits by a streptococcus. These authors attributed an etiologic rôle to the organism which they studied. Meyer ${ }^{4}$ made cultures from the throats of acute rheumatic fever patients directly into broth. The injection of this

* Submitted for publication Sept. 19, 1916.

* From the Medical Clinic, Presbyterian Hospital, Columbia University and the Rockefeller Institute Hospital, New York.

1. Achalme, P.: Compt. rend. Soc. de biol., 1891, Ninth Series, 3, 651.

2. Singer, G. : Aetologie u. Klinik d. acuten Gelenkrheumatismus, Vienna and Leipzig, 1898.

3. Westphal, Wassermann and Malkoff: Berl. klin. Wchnschr., 1899, 36, 638 .

4. Meyer, Fr.: Verhandl. d. 19 Cong. f. inn. Med., 1901, p. 542. 
mixed culture into rabbits was followed by a nonsuppurative arthritis ; 10 per cent. of the animals had pericarditis or pleurisy and one fourth of the animals showed a verrucous endocarditis. From all of these lesions a diplostreptococcus which clouded broth could be isolated. The joint exudates soon became sterile and the animal recovered without permanent injury to the joint. Because cultures from normal throats, or from the angina of other disease, produced either suppurative arthritis, fatal septicemia, or no lesions at all, Meyer ascribed a specific etiologic rôle to these streptococci.

Menzer, ${ }^{5}$ while confirming Meyer's results with streptococci from rheumatic angina, on the other hand was able to produce a similar arthritis and endocarditis with cultures from nonrheumatic or normal tonsils. He was unable to determine any colony, cultural or staining peculiarities in the streptococci from the tonsils of rheumatic patients compared with those from the tonsils of normal or nonrheumatic persons. He therefore concluded that "acute articular rheumatism is a peculiar clinical picture, not because there is a specific etiologic agent, but because the patient under certain inherited or acquired conditions and under acute or chronic influences reacts to the infection with common micro-organisms, apparently the organism found in the mouth, in a specific manner with manifestations which appear clinically as a specific illness."

Unfortunately, in none of these studies was any attention given to the hemolytic properties of the streptococci, so it is impossible to correlate absolutely the results with those of later workers. The evidence brought forward by $\mathrm{Cole}^{6}$ and by Harris ${ }^{7}$ to show that any streptococcus may produce destructive lesions of the joints likewise does not clear up this point, because the organisms studied were not divided into hemolytic and nonhemolytic forms. From our own studies and a review of the literature it is evident that the hemolytic forms may produce acute suppurative and destructive arthritis, provided they are not sufficiently virulent or injected in large enough doses to cause a fatal septicemia.

The work of $\mathrm{Cecil}^{8}$ in this laboratory, however, conclusively demonstrates that destructive lesions of the articular surfaces often follow the intravenous injection of Streptococcus viridans into rabbits. The character of the exudate in this series was mucoid or mucopurulent. The exudate in the arthritis produced by hemolytic streptococci is frankly purulent.

5. Menzer, A.: Die Aetologie des acuten Gelenkrheumatismus, Berlin, 1902.

6. Cole, R.: Jour. Infect. Dis., 1904, 1, 714.

7. Harris, N.: Tr. Chicago Path. Soc., 1905, 6, 303.

8. Cecil, R.: Jour. Exper. Med., 1916, 24, No. 6. 
In 1900 Poynton and Paine" gave the specific name "Diplococcus rheumaticus" to a diplostreptococcus which they isolated from four pericardial exudates, two blood cultures, two valve lesions and one angina, all from patients with acute rheumatic fever. Because they could demonstrate microscopically these organisms in theumatic lesions, and produce similar lesions in experimental animals with pure cultures, they felt justified in applying a specific name to them. So thoroughly imbued are these authors with the specific nature of their diplococcus that they ${ }^{10}$ apply the adjectives "rheumatic malignant" to the disease condition usually recognized as subacute streptococcus endocarditis. This they do because of the similarity of the micrococci associated with the two conditions. It is thus evident that their Diplococcus rheumaticus is similar to the Streptococcus viridans or mitis. Their cultural studies show that their organism is a diplostreptococcus giving a brownish color on blood agar plates, a turbidity in broth, and is gram-positive, though giving up the Gram stain more readily than do other gram-positive bacteria. The only point of difference from the ordinary Streptococcus viridans is in the size of the individual cocci, which they give as 0.5 microns. It is probable that the organisms measured by them were unusually small, such as are not infrequently encountered.

Another view of the rôle of streptococci in acute rheumatism is that of Rosenow ${ }^{11}$ that the streptococci are constantly undergoing changes, assuming and discarding elective affinities for specific structures. It will be seen that this is the direct antithesis of Menzer's opinion, which places the onus on the patient's susceptibility to infection with mouth streptococci. This incomplete review of the literature gives a fair idea of the various opinions of the relation of streptococci to acute rheumatic fever.

While none of the classifications of streptococci are absolutely satisfactory, that proposed by Schottmüller ${ }^{12}$ is almost universally accepted. In it we have hemolytic and nonhemolytic forms and, under the last group, those which form methemoglobin from the hemoglobin -the so-called viridans-and those which do not affect the hemoglobin, called anhemolyticus or saprophyticus by Le Blanc. ${ }^{13}$

A more extensive classification has been made by Andrewes and Horder, ${ }^{14}$ based on the fermentation reactions of the streptococci.

9. Poynton, F. J., and Paine, A.: Complete works collected in Researches on Rheumatism, London, 1913, p. 109.

10. Poynton, F. J., and Paine, A.: Complete works collected in Researches on Rheumatism, 1913, p. 312.

11. Rosenow, E. C.: Jour. Am. Med. Assn., 1915, 65, 1687.

- 12. Schottmüller, H.: München. med. Wchnschr., 1903, 50, 849.

13. Le Blanc: Centralbl. f. Bakteriol., Orig., 1911, 61, 68.

14. Andrewes, F. W., and Horder, T. J.: Lailcet, London, 1906, 2, 708, 775, 852. 
These authors recognized types which were associated to a certain extent with distribution in nature and pathogenic properties. While this classification has not been usually accepted, the study of fermentative reactions is of value and helps in a general way to place the organisms in groups.

In reviewing the various biochemical studies of streptococci, one occasionally meets with the results of studies made on organisms which have been isolated from patients with acute rheumatism. In trying to correlate these results one is struck with the diversity in types reported. Thus Andrewes and Horder $^{14}$ report that in the necropsies of fatal cases they secured two different main types, namely, Streptococcus pyogenes twice and anginosus variants seven times. They consider that these organisms may have been agonal invaders, because streptococci were only occasionally recovered from fatal cases. In testing a streptococcus rheumaticus from Poynton and Paine they found it to fall into the salivarius group and a Micrococcus rheumaticus sent by Beattie fell into the fecalis group. Beattie and Yates ${ }^{15}$ report that three different streptococci isolated from the synovial membrane of the knee joint of acute rheumatic patients fell into the fecalis group. Floyd and Wohlbach ${ }^{16}$ found two hemolytic streptococci, one, which clotted milk, fermented raffinose and salicin, hence fell into the Streptococcus anginosis group; the other did not change milk or the test sugars except lactose and fell into the pyogenes group. Still another one was nonhemolytic and fermented none of the sugars. Hopkins and Lang ${ }^{17}$ found one nonhemolytic streptococcus which did not ferment any of the test objects. Lyal1 ${ }^{13}$ reported on five streptococci, three isolated from the blood of rheumatics and two from the joints. All were indifferent to blood; one did not ferment either raffinose, intulin, salicin or mannite; one fermented only salicin, and three fermented salicin and mannite, which is characteristic of fecalis types. This small list proves that no single type of streptococcus has been constantly isolated in cases of acute rheumatic fever. Rosenow. ${ }^{19}$ in reporting on the etiology of acute rheumatism, states that he was able to isolate three different types of organisms from the joint exudates. Tested on blood agar plates, they were, first, a green type forming long chains; second, a type giving slight hazy hemolysis about the colonies and forming short chains; all of Type 2 were mannite fermenters; third, a type with small grayish colonies indifferent to blood. In this paper Rosenow reported on his method of isolating

15. Beattie, J. M., and Yates, A. G.: Jour. Path. and Bacteriol., 1911, 16, 247.

16. Floyd, C., and Wohlbach, S. B.: Jour. Med. Research, 1913, 29, 493.

17. Hopkins, J. A., and Lang, A.: Jour. Infect. Dis., 1914, 15, 63.

18. Lyall, H. W.: Jour. Med. Research, 1914, 30, 487.

19. Rosenow, E. C.: Jour. Infect. Dis., 1914, 14, 61. 
the organisms from joint fluid by mixing the fluid in deep tubes of ascites dextrose agar, and from the blood by placing the sediment of centrifugalized laked blood in a similar medium. In this way streptococci were obtained from the joint fluid of fourteen out of sixteen patients; from the blood in three out of four.

We have studied the type of streptococci which we have been able to isolate from the blood of patients with acute rheumatic fever. In all fifty-eight cases have been studied. Nine were studied at the Hospital of the Rockefeller Institute three years ago and the remainder have been from the wards of the Presbyterian Hospital during the past two years. All were typical cases, showing fever and polyarthritis, and responded in the characteristic manner to salicylates. Blood cultures were made shortly after the admission of the patients to the hospital and before salicylates had been administered; also afterward at such times as complications or relapses appeared. The cultures were made from a few hours to several days after the appearance of the arthritis. The cultures of joint fluids were made at the same time, but in many of the cases it was difficult to obtain fluid from the affected joints. For this reason there were fewer cultures of joint exudates. The joints aspirated were knee, ankle, elbow and wrist.

Cultural Methods: In every case blood cultures were made as follows: (1) of from 2 to 3 c.c. of blood in dextrose agar plates; (2) by placing from 5 to 10 c.c. of blood in 125 c.c. of 1 per cent. dextrose broth; (3) by laking 5 c.c. of blood in 50 c.c. of sterile distilled water, centrifugalizing and placing the sediment in deep tubes of ascites dextrose agar. The cultures were usually made in duplicate. In addition, in many instances, laked sediment was placed on the surface of blood agar slants and in ascites dextrose broth.

In making cultures of joint fluid, deep tubes of ascites dextrose agar were mixed with increasing amounts of the fluid, in most cases $1,2,3$, and 4 c.c. of fluid were placed in the different tubes. If there was sufficient fluid it was also put into deep tubes of ascitic dextrose broth and on the surface of blood agar slants. In this way all degrees of oxygen tension were obtained. The results are summarized in Table 1 .

Altogether eighty-five blood cultures were made on fifty-eight patients with seven positive results, a percentage of 8.3 . In one instance the cultures $59 F$ and $B 38$ were obtained from the same patient, so that in only six patients were positive cultures obtained. With the exception of this case, positive cultures were obtained only once in each patient and repetition of the cultures a short time afterward gave negative results. In three instances the cultures were 
obtained at the time of an acute pericarditis. In two of these $A 49$ and $38 \mathrm{D}$, adults, previous cultures had been sterile, but at the time of the appearance of the pericarditis positive cultures were obtained, while later cultures were sterile. The other case of pericarditis was that of a child, $59 \mathrm{~F}$, admitted to the Rockefeller Hospital in 1914. The culture was positive at the time of admission, but negative later. In 1916 she was readmitted to that hospital with typical acute rheumatism and polyarthritis; culture $B 38$ was obtained, but repeated attempts to secure a confirmation resulted in sterile cultures. Culture $B 39$ was from a child and $A 65$ and $A 135$ from adults with typical acute rheumatic fever.

All of the cultures of joint exudates have been sterile. Joints have been aspirated within four hours after the appearance of the exudate and at various later intervals. As positive blood cultures have been obtained from patients at the time that joint exudate c11-

TABLE 1.--Results of Blood and Joint Cultures

\begin{tabular}{|c|c|c|c|c|c|c|}
\hline & \multicolumn{3}{|c|}{ Blood Cultures } & \multicolumn{3}{|c|}{ Joint Cultures } \\
\hline & $\begin{array}{c}\text { Number } \\
\text { of } \\
\text { Patients }\end{array}$ & $\begin{array}{c}\text { Number } \\
\text { of } \\
\text { Cultures }\end{array}$ & $\begin{array}{l}\text { Number of } \\
\text { Positive } \\
\text { Cultures }\end{array}$ & $\begin{array}{c}\text { Number } \\
\text { of } \\
\text { Patients }\end{array}$ & $\begin{array}{c}\text { Number } \\
\text { of } \\
\text { Cultures }\end{array}$ & $\begin{array}{l}\text { Number of } \\
\text { Positive } \\
\text { Cultures }\end{array}$ \\
\hline Adults........... & 44 & 60 & 4 & 23 & 31 & 0 \\
\hline Children......... & 14 & 25 & 3 & 2 & 3 & 0 \\
\hline Total....... & 58 & 85 & $7(8.3 \%)$ & 25 & 34 & 0 \\
\hline
\end{tabular}

tures were sterile, it is evident that the medium was suitable for the growth of the streptococci. We therefore do not feel that the difference between our results and those of Rosenow can be explained by technical errors, such as age of the arthritis or faults with culture mediums. In addition all of the joint exudates have been examined microscopically and in no instance have we found diplococci or streptococci in smears.

Patients have also been studied in whom arthritis was not present. These include principally cases of endocarditis with fever and accompanying conditions, such as pleurisy, pericarditis or chorea. While it is generally considered that these are rheumatic manifestations, it was thought better to place them under a separate heading. In two such instances streptococci were obtained in blood culture. Culture $A 119$ was obtained from a patient during a febrile attack accompanied by pain in the knee, but no other arthritis symptoms. Culture $A 141$ was obtained from a child with pericarditis and endocarditis during an attack of "decompensation" and pleurisy. Subsequent cultures were 
sterile, but the "rheumatic" nature of the condition was demonstrated a year later at necropsy. In addition, three cases of acute rheumatic pericarditis and two of endocarditis gave sterile blood cultures during the acute period of the disease. The aspirated pericardial fluid from one of the pericarditis patients also yielded negative cultures.

Necropsies: The difficulty of obtaining a pure culture at necropsy is almost insurmountable and hence the results of necropsy cultures are inconclusive. Six fatal 'cases have been studied. Three of these patients had acute pericarditis and acute endocarditis, two acute endocarditis and one chorea and endocarditis with terminal pneumonia.

TABLE 2.-REsumé of Positive Cultures

\begin{tabular}{|c|c|c|c|c|}
\hline \multirow{2}{*}{ Number } & \multicolumn{2}{|c|}{$\begin{array}{c}\text { Day of Disease after } \\
\text { Onset of }\end{array}$} & \multirow{2}{*}{$\underset{\text { Cultures Grew* }}{\text { Mediums in Which Positive }}$} & \multirow{2}{*}{$\begin{array}{l}\text { Joint } \\
\text { Cultures }\end{array}$} \\
\hline & Arthritis & Pericarditis & & \\
\hline$A 6 \overline{5}$ & 4 th & 0 & $\begin{array}{l}\text { Laked sediment in aseites dextrose } \\
\text { agar tube only }\end{array}$ & Sterile \\
\hline$A 135$ & 11th & 0 & All mediums.............................. & Sterile \\
\hline B 39 & 4 th & 0 & Two broth flasks, no others made....... & Not made \\
\hline $29 \mathrm{~F}$ & 1st attack 9 th & 1st & One broth flask only................... & Not made \\
\hline B 38 & $2 d$ attack $2 d$ & $\cdots$ & All mediums; 100 colonies per c.c. ....... & Sterile \\
\hline \multirow{2}{*}{$38 \mathrm{D}$} & 12 th & $\cdots$ & Sterile & \\
\hline & 15 th & 1st & Laked sediment in ascitic broth only.... & Sterile \\
\hline \multirow{2}{*}{ A 49} & 14th & $\cdots$ & Sterile & \\
\hline & 19 th & 1st & $\begin{array}{l}\text { Colonies in only } 1 \text { dextrose blood agar } \\
\text { plate }\end{array}$ & Not made \\
\hline A 119 & $\begin{array}{l}\text { Indeterminate } \\
2 \text { day of feb- } \\
\text { rile attack }\end{array}$ & $\begin{array}{l}\text { Previous } \\
\text { attack }\end{array}$ & $\begin{array}{l}\text { Colonies in only } 1 \text { dextrose blood agar } \\
\text { plate }\end{array}$ & Not made \\
\hline A 141 & $\begin{array}{l}\text { Indeterminate } \\
\text { during pleu- } \\
\text { risy }\end{array}$ & $\begin{array}{l}\text { Previous } \\
\text { attack }\end{array}$ & $\begin{array}{l}\text { Laked sediment in } 1 \text { ascitic dextrose } \\
\text { agar tube only }\end{array}$ & Not made \\
\hline A 17 & Necropsy & $21 / 2 \mathrm{mo}$. & Blood agar plates & \\
\hline
\end{tabular}

* Positive cultures developed in all mediums in two cases; laked sediment in deep ascitic dextrose agar tubes only, in two cases; laked sediment in ascitic broth only, one case; blood broth flask only, one case; both blood broth flasks, one case; dextrose blood agar plates only, two cases.

From the vegetations of one of the endocarditis cases streptococci were grown in pure culture and from the other a mixed culture of staphylococci and diphtheroid bacilli. From the heart's blood of the chorea and endocarditis patient, a Group 4 pneumococcus was obtained. In the cases of pericarditis and endocarditis the cultures from endocardial lesions in one case yielded a staphylococcus and a green-forming streptococcus which fermented raffinose. From the second case, in which the heart was opened under rigid precaution after removal from the necropsy room, three types of cocci were 
obtained, (1) pneumococcus, (2) hemolytic streptococcus, and (3) a green-forming streptococcus. The streptococci from both these cases were lost before they were completely studied. In the third case $A 179$, diphtheroid bacilli and green streptococci were obtained from all the diseased valves. The characteristics of these cocci are given under the subsequent studies. The case reports are given at the end of the paper.

Cultural Characteristics. - The cultural characteristics and virulence of the organisms have been studied immediately on isolation. All were gram-positive cocci, with a tendency to grow in pairs or short chains. Plain broth cultures were turbid and did not clear on the

TABlE 3.-Cultural and Virulence Characteristics

\begin{tabular}{|c|c|c|c|c|c|c|c|c|c|c|c|c|}
\hline \multirow{2}{*}{\multicolumn{2}{|c|}{ No. }} & \multicolumn{2}{|c|}{ Growth in } & \multirow{2}{*}{$\begin{array}{l}\text { Bile Solu- } \\
\text { bility }\end{array}$} & \multirow{2}{*}{$\begin{array}{l}\text { Effect } \\
\text { on Red } \\
\text { Blood } \\
\text { Cells }\end{array}$} & \multicolumn{5}{|c|}{ Fermentation Reactions } & \multirow{2}{*}{\multicolumn{2}{|c|}{$\begin{array}{l}\text { Lethal } \\
\text { Dose for } \\
\text { White } \\
\text { Mice }\end{array}$}} \\
\hline & & $\begin{array}{l}\text { Bouil- } \\
\text { lon }\end{array}$ & $\begin{array}{l}\text { Ascitic Dex- } \\
\text { trose Agar }\end{array}$ & & & Milk & $\begin{array}{l}\text { Raff- } \\
\text { nose }\end{array}$ & $\operatorname{Inu}_{\text {lin }}$ & $\underset{\text { cin }}{\text { Sali- }}$ & $\begin{array}{l}\text { Man- } \\
\text { nite }\end{array}$ & & \\
\hline B & 38 & Turbid & $\begin{array}{l}\text { Marked } \\
\text { clouding }\end{array}$ & Insoluble & Green & + & + & + & 0 & 0 & 2 c.c. & 0 \\
\hline A & 179 & Turbid & $\begin{array}{l}\text { Marked } \\
\text { clouding }\end{array}$ & Insoluble & Green & + & 0 & 0 & 0 & 0 & 2 c.c. & + \\
\hline B & 39 & Turbid & $\begin{array}{l}\text { Marked } \\
\text { clouding }\end{array}$ & Insoluble & Green & + & 0 & 0 & 0 & 0 & 2 c.c. & 0 \\
\hline 59 & $\mathbf{F}$ & Turbid & $\begin{array}{l}\text { Marked } \\
\text { clouding }\end{array}$ & Insoluble & Green & + & 0 & 0 & 0 & 0 & 0.1 c.c. & + \\
\hline A & 49 & Turbid & $\begin{array}{l}\text { Marked } \\
\text { clouding }\end{array}$ & Insoluble & Green & + & 0 & 0 & 0 & 0 & 0.1 c.c. & + \\
\hline A & 141 & Turbid & $\begin{array}{l}\text { Marked } \\
\text { clouding }\end{array}$ & Insoluble & Green & + & 0 & 0 & 0 & 0 & 2 c.c. & + \\
\hline$A$ & 219 & $\begin{array}{c}\text { Slightly } \\
\text { turbid }\end{array}$ & $\begin{array}{l}\text { Marked } \\
\text { clouding }\end{array}$ & Insoluble & $\begin{array}{c}\text { Sitghtiy } \\
\text { green }\end{array}$ & + & 0 & + & + & 0 & 2 c.c. & 0 \\
\hline 38 & $\mathrm{D}$ & $\begin{array}{l}\text { Slightly } \\
\text { turbid }\end{array}$ & $\begin{array}{l}\text { Marked } \\
\text { clouding }\end{array}$ & Insoluble & Green & + & 0 & 0 & + & 0 & 1 c.c. & + \\
\hline$A$ & 135 & Turbid & $\begin{array}{l}\text { Marked } \\
\text { clouding }\end{array}$ & Insoluble & Green & + & 0 & 0 & + & + & 2 c.c. & 0 \\
\hline$A$ & 65 & Turbid & $\begin{array}{l}\text { Marked } \\
\text { clouding }\end{array}$ & Insoluble & Green & + & + & 0 & 0 & 0 & 0.1 c.c. & + \\
\hline
\end{tabular}

addition of equal parts of ox bile. No capsules could be demonstrated in the smears of the peritoneal exudates of mice inoculated intraperitoneally with the organism. The action on blood was studied, both on human blood plain agar plates and by mixing diminishing dilutions of twenty-four hour plain broth cultures with equal parts of 5 per cent. suspension of sheep's blood cells and incubating two hours. None of the strains produced hemolysis of the cells or clearing about the colonies on the blood agar plate and all turned the cells brown and gave a green appearance on the plates. This is characteristic of the so-called Streptococcus viridans. 
The fermentation reactions were studied with milk, raffinose, inulin, salicin and mannite in Hiss serum water mediums. These mediums were heavily inoculated with an actively growing twenty-four-hour broth culture, and observed over a period of ten days. The fermentation reactions were studied at the time of isolation of the culture, and again in June, 1916. None of this series underwent any change during the interval. All the strains produced acid and clot in milk. Five of the strains did not ferment any of the test objects. The other five showed individual fermentative capacity, no two falling into the same group. Only one was a mannite fermenter, thus placing only one of our series in the fecalis group.

The virulence was tested by injecting 2 c.c. 1 c.c. and 0.1 c.c. of a twenty-four-hour plain broth culture intraperitoneally into white mice. The lethal dose of three of the strains was 0.1 c.c., of one it was 1 c.c., of two it was 2 c.c., and four were not fatal in 2 c.c. quantities. Only in the point of virulence have organisms in this group shown peculiarities. Compared with green-producing strepto= cocci from normal mouths or from patients with subacute bacterial endocarditis most of these strains have been distinctly virulent, although compared with hemolytic streptococci or pneumococci, their virulence is low. On cultivation the original virulence has been fairly well maintained, and repeated passage through mice has failed to increase the virulence to any appreciable degree.

Immunologic Classification.-In addition to a study of the cultural characteristics, an attempt has been made to determine whether there was any immunologic grouping. For this purpose the complement fixation reaction has been used, employing antiformin solutions of the various streptococci as antigens. The exact method is fully described elsewhere. ${ }^{20}$ Rabbits were injected intravenously with killed streptococci until their serum gave good fixation with its homologous antigen. It was then tested for its complement fixing power against antigens prepared from all the streptococci. The results are summarized in Table 4.

It will be seen that no single group exists. One member, $A 65$, is not related to any of the others. Among the remaining strains roughly three groups can be made out. The first consists of three strains, $B$ 38, $A 179$ and $B 39$; the second of $59 F$ and $A 49$, which appear to be almost identical; and a third, which consists of $A 141, A 119$ and $38 D$. The first and second groups are connected by serum $A 141$, which also fixes antigen $A 135$. Comparing the individual strains, however,

20. Kinsella, R. A., and Swift, H. F.: A Classification of Nonhemolytic Streptococci. To be published. 
we find that the only two which appear to be very closely related are $59 F$ and $A 49$.

It is not the intention to discuss the general subject of the classification of streptococci by means of the complement fixation reaction, as that is more fully considered in another communication. The evidence brought forward in this table is merely to show the immunologic relationship of the various strains.

A comparison of the fixing properties with the fermentation reactions and virulence of the several strains shows that no strict relationship exists between the various fermentation groups and the groups as determined by complement fixation. In the study of the virulence of the strains no special tendency toward virulence is noted among any one of the various fermentation groups or complement fixation groups,

TABle 4.-Grouping by Means of Complement Frxation Reactions*

\begin{tabular}{|c|c|c|c|c|c|c|c|c|c|c|}
\hline \multirow{2}{*}{$\begin{array}{l}\text { Serum of Rabbits } \\
\text { Immunized With }\end{array}$} & \multicolumn{10}{|c|}{ Antigens } \\
\hline & B 38 & A 179 & B 39 & $59 \mathrm{~F}$ & $\overline{A 49}$ & A 141 & A 119 & $38 \mathrm{D}$ & A 135 & $A 05$ \\
\hline B $38 \ldots \ldots$. & + & - & \pm & 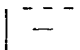 & - & - & - & - & - & - \\
\hline A $179 \ldots$ & - & + & \pm & - & - & - & - & - & - & - \\
\hline $\mathrm{B} 39 \ldots$. & + & + & + & + & \pm & - & - & - & - & - \\
\hline $59 \mathrm{~F} . . .$. & - & - & - & + & + & - & - & - & - & - \\
\hline A $49 . \ldots \ldots$ & \pm & \pm & - & + & + & - & - & - & - & - \\
\hline A $141 \ldots \ldots \ldots \ldots \ldots$ & - & - & - & + & + & + & + & + & + & - \\
\hline A $119 . \ldots \ldots \ldots \ldots \ldots$ & - & - & - & - & - & - & + & + & - & - \\
\hline $38 \mathrm{D} \ldots \ldots \ldots \ldots \ldots \ldots$ & - & - & - & - & - & - & - & + & - & - \\
\hline A $185 \ldots \ldots \ldots \ldots \ldots$ & - & - & - & - & - & - & - & - & + & - \\
\hline A $65 \ldots \ldots \ldots \ldots \ldots \ldots$ & - & - & - & - & - & - & - & - & - & + \\
\hline
\end{tabular}

* In this table + signifles that flxation with heterologous antigen was of practically the same strength as with homologous antigen; \pm signifles that flxation with heterologous antigen was weaker than with homologous antigen; - signifles negative reaction.

with the exception of $59 F$ and $A 49$, which seem to be closely related in all their characteristics. Compared with a larger number of greenforming streptococci obtained from blood cultures of Streptococcus viridans from endocarditis patients and from the throats of acute angina patients, the streptococci from acute rheumatic fever patients could not be differentiated by means of cultural characteristics. In the immunologic studies most of the streptococci from rheumatic fever patients fell in one side of the large group, although various individual members are scattered throughout the group. In the matter of virulence six out of ten rheumatic fever strains were virulent, while with one exception all the endocarditis strains were avirulent. On the other hand, angina strains were often virulent to 1 c.c. or less. 
Study of Antibodies in Patients' Serum.-After it was shown ${ }^{21}$ that the serum of patients with subacute streptococcus endocarditis contained antibodies against homologous strains, it was thought that aritibodies might be present in the serum of acute rheumatic fever patients whose blood yielded a positive culture. Likewise, if a specific "Streptococcus rheumaticus" existed, the serums of rheumatic fever patients might show specific antibodies. These antibodies were tested for by means of the thread reaction, a modification of the agglutination reaction which has proved more satisfactory with streptococci than the ordinary agglutination reaction. The serums of five patients tested failed to react to their respective streptococci. The serums of twelve other rheumatic fever patients failed to react with any of the four strains, $A 49,59 F, 38 D$ and $A 65$, a result which is easily explained by the individuality of these strains. The failure of serums to react with homologous strains is, however, most striking when compared with the results obtained in a type of disease such as subacute streptococcus endocarditis, in which we are certain of the relationship between the bacterium and the disease.

\section{COM MENT}

We do not feel that our results clear up the much-vexed question of the relation of the nonhemolytic streptococcus to acute rheumatic fever. From a statistical point of view the small percentage of positive cultures would incline toward the negative side of the question. Streptococci are found in the blood of scarlet fever patients with an equal frequency, and yet most observers do not believe that the streptococcus is the cause of scarlet fever, but rather that this organism acts as a secondary invader; and it is conceivable that it may play a similar rôle in acute rheumatic fever. The relatively high frequency with which the streptococcus was demonstrated in patients with complications such as pericarditis or pleurisy lends support to this opinion. Again, the failure to demonstrate constantly the streptococcus in the verrucae of acute rheumatic endocarditis is an argument against the specific etiologic relationship of this organism.

On the other hand, a small proportion of positive cultures may simply mean that the streptococci circulate in the blood stream only for short periods, or are rapidly killed off, so that the methods employed are sufficient to demonstrate them only occasionally. Furthermore, our studies of the cultural and immunologic characteristics show that the streptococci which are present in this disease are merely various members of the Streptococcus viridans group, and should not be separately classified as Streptococcus rheumaticus. Of extremely

21. Kinsella, R. A.: The Archives Int. Med., this issue, p. 367. 
important bearing on this question are the findings in cultures $59 \mathrm{~F}$ and $B$ 38. At the time of the first attack, streptococcus $59 F$ was isolated. After a prolonged course the patient recovered from every evidence of acute or subacute infection. After a year of good health she suffered a relapse, or as is more probable, a new infection. At this time streptococcus $B 38$ was recovered from her blood. This proved to be an organism that differed culturally, immunologically and in its virulence from the strain originally isolated. In addition, no complement fixing or agglutinating antibodies against $59 \mathrm{~F}$ could be demonstrated in the patient's blood at the time of the second infection. If the infection was a chronic one due to streptococcus $59 \mathrm{~F}$, we would expect to find antibodies such as may be demonstrated in the serum from cases of subacute streptococcus endocarditis. We feel that more detailed observation and experimentation is required to decide the points at present under discussion.

Another point should be mentioned: There is a tendency among some workers to consider that because a green streptococcus is isolated from the blood of a patient with endocarditis, there is sufficient ground for classifying the case in question as subacute bacterial endocarditis - the type so well described by Libman. This disease, with its characteristic symptomatology, is now generally recognized as a fatal malady. From our studies we feel that the essential factors in its recognition do not depend alone on the isolation of the infecting organism, but on such characteristic symptoms and signs as petechiae, embolic lesions and progressive downward course, in addition to the picture of an endocarditis. The findings in our cases indicate that green streptococci can be isolated from the blood stream, and still the patient may not suffer from subacute bacterial endocarditis. Some of the patients have been followed for a year or more without showing any signs of this disease, and case $A 141$, a year after yielding a single positive blood culture, showed at necropsy characteristic rheumatic lesions in the heart valves and muscle.

\section{SUMMARY AND CONCLUSIONS}

1. Cultures of the exudate aspirated from the joints in acute rheumatic arthritis have been uniformly sterile.

2. Nonhemolytic streptococci have been recovered in blood culture from less than 10 per cent. of patients suffering from acute rheumatic fever.

3. Similar streptococci have been recovered from the active endocardial lesions in only half of the fatal cases of acute rheumatic fever.

4. From the above results it seems evident that no type of streptococcus has been constantly associated with acute rheumatic fever. 
5 . We do not feel that the etiologic relationship between the streptococcus and acute rheumatic fever has been definitely proved, but if the streptococcus is the etiologic agent in acute rheumatic fever, it is shown by means of cultural and immunologic studies that it is through various members of the viridans group, and hence no one member can be called the Streptococcus rheumaticus.

\section{CASE REPORTS}

$A$ 65.-J. C., a man aged 19, a painter, was admitted to the Presbyterian Hospital Jan. 8, 1915, and discharged February 7. On January 9, herniotomy was performed. The temperature was normal four days and then it ranged between 99 and 103 for seven days. On the 19th blood cultures made in broth flasks were sterile. On the 25 th the patient suffered pain in both ankles and the right knee. On the 27 th there were general malaise and chilly sensations, the joints being swollen and tender. Blood culture in broth flasks was sterile. On the 28th blood cultures in broth flasks and ascitic dextrose agar plates were sterile. On the 29 th blood cultures were positive for $A 65$. Cultures were developed only in laked sediment in ascitic dextrose agar tube. A culture of fluid from the right knee was sterile. On the 31 st extension of arthritis to wrist and hands followed. Prompt relief was obtained from 100 grains of sodium salicylate per day. On February 7 the patient insisted on going home because he felt so well. The serum gave no thread reaction with $A 65$ culture.

$A$ 135.-O. C., a man aged 26, a tile-layer, was admitted to the Presbyterian Hospital April 7, 1915, and discharged on May 11. He had suffered six years before with "acute rheumatism," involving all the joints. He was ill four months five years before with a similar attack, his illness lasting one month. His present illness began two weeks before with "cold in head and chest." Eleven days before his left knee became swollen, tender and painful; this was followed by the involvement of all the joints of the arms and hands and the right knee. There was very little general illness. Physical examination revealed pyorrhea. There was an abscess at the root of the first right lower molar. The heart was not enlarged, but there was a faint murmur at the apex not transmitted and at the base. There was general polyarthritis. On April 7 a blood culture proved positive for $A$ 135. Growth took place in all mediums. The right knee yielded 20 c.c. of murky, lemon-colored fluid, the left 15 c.c. of similar fluid. Cultures of this fluid were sterile. Fever was absent after three days of salicylates, and the joints returned to normal after five days. The patient was discharged without any complications of the disease.

$B$ 39.-B. M., a girl, aged 6 years, was admitted to the Presbyterian Hospital March 10, 1916, and discharged April 9. The family history showed that the mother and one sister had syphilis. The child had had some joint trouble when a few weeks old. She had acute rheumatism eighteen months before admission and numerous attacks of tonsillitis, the last being one month before. Three days before there was pain in both wrists, lasting two days, and one day before the ankles became painful and swollen. Physical exansination showed the tonsils much enlarged, not acutely inflamed. The heart was enlarged to the left and downward. There was a systolic murmur at the apex. A diastolic murmur was perceptible over the aortic area. Both ankles were hot, terder and swollen. On March 11 a blood culture in two flasks showed both positive for $B 39$. On the 14th a blood culture proved sterile. The arthritis cleared up without salicylates. On April 7 a blood culture was sterile and the general condition was good.

$59 \mathrm{~F}$ and $B$ 38.-F. B., a schoolgirl, aged 8 years, was admitted to the Rockefeller Institute Hospital May 26, 1914, and discharged June 2, 1915. The child was suffering with rheumatic fever, acute pericarditis, valvular disturbance and mitral insufficiency. She complained of pain over the heart. Two weeks previously the mother and one brother of the patient had sore throat, but the patient did 
not. The patient had measles at 4 , but no scarlet fever. She often had bronchitis, but no sore throat. At 1 year there was twitching of the left arm (chorea). She never had pain in the joints. Eleven days previously the patient fell while skating. Ten days before she vomited and had headaches. Nine days before her feet hurt and the right one was swollen. Eight days before both ankles and knees were swollen and painful. This gradually decreased. Her general condition improved from day to day. The day before entrance she was out of bed, and on the day of entrance she was attacked with a sudden pain in the region of the heart. Physical examination revealed dyspnea, painful expression and a dry cough. There was a friction rub over heart. The heart was $4 \mathrm{~cm}$. to the right and 11 to the left of the midsternal line. The liver was enlarged, extended $3 \mathrm{~cm}$. below the costal margin and was tender. There was no edema. The joints were negative. Blood culture was positive for $59 \mathrm{~F}$. Growth took place only in the broth flask. The friction rub persisted until June 8. Fever lasted until July 12. From.September 21 to 29 there was fever, but the blood cultures were sterile. On Aug. 7, 1915, the tonsils were removed.

The patient was readmitted to the hospital March 9, 1916. She had remained well until one month before, when she had grip and fever for three days. Three weeks before she had grip for two weeks, with high fever; then she was well one week. Two days before entrance she vomited and had headache. The day before she had pain in both ankles. Examination showed no petechiae. The throat was clear. The heart was enlarged, and there was an apical systolic murmur. There was heat and tenderness in the left ankle. A blood culture was positive for $B 38$, giving 100 colonies per cubic centimeter, which developed in all mediums. February 12 both knees were swollen and tender. March 10 the ankles, knees, elbows and wrists had been involved. Blood cultures on March 11,12 and 13 were sterile. On the 12 th 5 c.c. turbid fluid were aspirated from the knee joint, but a culture proved sterile. There was no trace of petechiae. The fever ranged from 101 to 102 until May 5.

38 D.-J. McK., a blacksmith, aged 58, was admitted to the Rockefeller Institute Hospital March 15, 1914, and discharged June 9, 1914. There was a history of acute rheumatism four years previously. There had been no cardiac symptoms afterward. Two weeks before entrance he caught cold, and eleven days before his left wrist became red and swollen. Five days before his knees became involved. and four days before his right wrist also was affected. $\mathrm{He}$ was confined to bed for two days. Examination showed the heart to be slightly enlarged. There was an apical systolic murmur. There was acute arthritis in the right wrist, both elbows, the knees and the ankles. On March 16 a blood culture was sterile, and the fluid from both knees and ankles was sterile. On March 19 there was a pericardial friction rub. A blood culture was positive for $38 \mathrm{D}$, developing from laked sediment in ascitic broth. On March 20 a blood culture was sterile. On the 21 st there were signs of fluid in the left pleural cavity. On the 26th the pericardial rub had disappeared and the blood culture was sterile. From March 29 to April 11 there was continuous auricular fibrillation. On April 12 the heart showed sequential rhythm. May 9 the patient showed a slow but steady convalescence.

A 49.-A. G., a waitress, aged 23, was admitted to the Presbyterian Hospital Jan. 16, 1915, and discharged April 6, 1915. Eleven years before the patient had a severe acute articular rheumatism, followed by chorea. The present illness began two weeks before with polyarthritis, involving both arms and legs. Later this had receded, but there were fever and sweating, and the previous few days dyspnea. Examination showed the heart to be enlarged, and there was an apical systolic murmur. The left wrist was swollen, red and tender. On January 16 blood culture proved sterile. From the 17 th to the 19 th the wrists, shoulder and knees were involved. On January 21 there was a pericardial rub. ${ }^{22}$ Blood

22. During the period from Jan. 16 to 23,1915 , the patient was taking sodium salicylate from 100 to 200 grains per day. 
culture was positive for $A 49$, colonies arising in one blood agar plate. This was followed by effusion into the pericardium, which persisted until the middle of February. On February 1 she had a febrile attack without new symptoms, but a blood culture was sterile. On the $3 \mathrm{~d}$ there was a fresh effusion into the knee joint, but a culture was sterile. Convalescence was slow but steady until the patient was discharged April 1.

In the spring of 1916 the patient reported another attack of acute rheumatic fever without cardiac involvement. In May, 1916, the blood serum gave no complement fixation with antigen $A 49$.

$A$ 119.-H. F., a boy, aged 16 , a clerk by occupation, was admitted to the Presbyterian Hospital March 2, 1915, and discharged April 9, 1915. The boy had attacks of precordial pain and dyspnea in 1909, 1910, 1911 and 1912. In 1912 he had some joint pains. In 1913 in this hospital a diagnosis was made of acute simple endocarditis, fibrinous pericarditis and left hydrothorax. There were double murmurs in both mitral and aortic areas. The boy was well and working until two weeks before entrance. He had cough two weeks, with increasing dyspnea, swelling of abdomen and legs. A physical examination showed dyspnea, orthopnea, with the throat clear, except for enlarged tonsils. The heart was much enlarged to the right and left. There were double mitral and double aortic murmurs, congestion of the lungs, enlarged liver, ascites and edema of the legs. On March 5 the patient had an attack of pulmonary edema. On the 9th steady improvement was apparent. March 14 to 16 the temperature was 102 . There were precordial pain and pain in the left knee, but no swelling or redness.

- On March 16 a blood culture was positive for $A 119$, colonies developing on blood agar plate. There was immediate improvement after taking salicylates. April 5 the temperature was normal, and the patient showed steady improvement. A blood culture was then sterile. The boy was discharged April 9, and he returned to work.

A 141.-J. K., a schoolgirl, aged 10 years, was admitted to the Presbyterian Hospital Oct. 3, 1914, remaining till Dec. 29, 1914. She was readmitted April 7, 1915 , and remained till June 16, 1915. She had diphtheria at the age of 5 years. She was always subject to sore throat. In April, 1914, she suffered from dizziness and vomiting. A diagnosis of rheumatic heart was made. This cleared up with rest. On Oct. 3, 1914, there were dizziness, cyanosis and dyspnea for three days. Examination showed the heart to be enlarged. An apical systolic murmur was transmitted over the entire chest. There were congestion of the lungs and enlarged liver. Fever of 103 was present, with respiration 56. On October 7 a blood culture showed three plates sterile. On the 12 th a blood culture of two plates of dextrose blood agar was sterile. From the 14th to the 24th a precordial friction rub was perceptible. On the 29 th there was general edema. November 24 the patient showed steady improvement, but there was fever of 101 each day. A blood culture on four dextrose blood agar plates was sterile. On December 22 the patient was much improved and was discharged.

She was readmitted April 7, 1915, complaining of increasing anorexia, nausea, pain in the chest and coughing. The throat was clear. The heart was enlarged, and showed a systolic retraction over the precordium. There were systolic and presystolic murmurs at the apex. The lungs were congested and a friction rub over the right base posteriorly was discernible. On April 13 a blood culture was positive for $A$ 141, colonies developing only in laked sediment in ascites dextrose agar tube. On April 19 and May 20 blood cultures were sterile. June 1 the patient showed a steady convalescence, but she had a temperature of 101 nearly every day. On June 16, 1916, she was readmitted in extremis. A blood culture was sterile.

Necropsy showed adherent pericardium, acute vegetative endocarditis of verrucous type, chronic cardiac valvular disease, chronic mural endocarditis, adherent pericardium, pleuritic adhesions (both sides) and rheumatic myocarditis.

$A$ 179.-A. B., a schoolgirl, aged 12 years, was admitted to the Presbyterian Hospital Feb. 26, 1915, and died May 20, 1915. She had acute rheumatism four 
years before, lasting three weeks. Two weeks before entrance there was pain in the legs; then the shoulders, ankles and knees became involved. There was pain over the precordium for several days, increasing dyspnea and weakness. Examination showed the pharynx to be diffusely reddened and the tonsils large. The heart was enlarged to the left and downward. There was a distinct gallop rhythm, with systolic and diastolic murmurs at the apex. The liver was enlarged, and there was tenderness over it. The right hand, left ankle and right knee were tender, painful and swollen. February 27 a blood culture was sterile. On March 3 the area of cardiac flatness was much increased. The cardiohepatic angle was obtuse and the heart sounds muffled. From March 17 to April 12 the patient had attacks of slowing of the pulse in which the rate was one-half the ordinary rate. Electrocardiograms showed sudden alternating transition of auricular rate from 53 to 108 . At both rates the conduction mechanism was normal. From April 14 to 18 there were precordial friction rubs, and also from May 4 to 7 . On May 14 there was much precordial pain, with chorieform movements. May 20 the condition steadily grew worse until death.

Necropsy showed acute rheumatic endocarditis, involving the mitral, aortic and tricuspic valves and foramen ovale; acute pericarditis, adherent pericardium; rheumatic myocarditis; many Aschoff bodies; cardiac hypertrophy; rheumatic pleurisy; mediastinitis; petechial hemorrhages into the fatty tissue of the abdomen, the pectoralis minor muscles and the pericardium, and beneath the endocardium in the right auricle; acute hemorrhagic enteritis. Cultures showed the hemorrhagic area from the left pectoralis minor muscle to be sterile. The heart's blood showed three diphtheroid colonies. The valve lesions were. cultured and diphtheroid and green forming streptococci $A 179$ developed from each valve, including the tricuspid valve, the mitral valve and the aortic valve. 